\title{
Principles of aortic valve repair
}

\author{
Gebrine El Khoury, MD, and Laurent de Kerchove, MD
}

\begin{abstract}
During the past 15 years, important advances in the field of aortic valve repair have transformed it from an infrequent and anecdotal exercise to a feasible and attractive alternative to valve replacement in selected patients with pure aortic insufficiency. These advances include a deeper understanding of the functional anatomy of the aortic valve and pathophysiologic mechanisms of aortic insufficiency; the development of surgical techniques to restore normal geometry to the aortic root and to restore the cusp coaptation; and the development of a common terminology that can be used by all clinicians to describe the lesions, discuss repair techniques, and compare immediate and long-term outcomes after aortic valve repair. This article attempts to describe the important principles of aortic valve repair by focusing on functional anatomy, surgical techniques for cusp repair, and outcome. By analogy with mitral valve repair, we include in the term aortic valve repair not only the repair of the cusp but also the valve-sparing root replacement or any kind of functional aortic annulus stabilization. (J Thorac Cardiovasc Surg 2013;145:S26-9)
\end{abstract}

During the past 15 years, important advances in the field of aortic valve (AV) repair have transformed it from an infrequent and anecdotal exercise to a feasible and attractive alternative to valve replacement in selected patients with pure aortic insufficiency (AI). These advances include a deeper understanding of the functional anatomy of the AV and the pathophysiologic mechanisms of AI; the development of surgical techniques to restore normal geometry to the aortic root and to restore cusp coaptation; and the development of a common terminology that can be used by all clinicians to describe the lesions, discuss repair techniques, and compare immediate and long-term outcomes after AV repair. This article attempts to describe the important principles of AV repair by focusing on functional anatomy, surgical techniques for cusp repair, and outcome. By analogy with mitral valve repair, we include in the term $A V$ repair not only the repair of the cusp but also valve-sparing root replacement or any kind of functional aortic annulus stabilization.

\section{ANATOMY OF THE AV AND THE FUNCTIONAL AORTIC ANNULUS}

The AV leaflets insert into the aortic annulus proximally at the aortoventricular junction (AVJ) and distally at the sinotubular junction (STJ). As a functional entity, the AV consists of the STJ and the AVJ, which together form the

\footnotetext{
From the Divisions of Cardiothoracic and Vascular Surgery, Université Catholique de Louvain, Cliniques Universitaires Saint-Luc, Brussels, Belgium.

Disclosures: Drs El Khoury and de Kerchove have nothing to disclose with regard to commercial support.

Read at The American Association for Thoracic Surgery Aortic Symposium, New York, New York, April 26-27, 2012.

Received for publication May 30, 2012; revisions received Oct 12, 2012; accepted for publication Nov 28, 2012; available ahead of print Dec 20, 2012.

Address for reprints: Gebrine El Khoury, MD, Division of Cardiothoracic and Vascular Surgery, Cliniques Universitaires St-Luc, Ave Hippocrate 10, 1200 Brussels, Belgium (E-mail: gebrine.elkhoury@uclouvain.be).

0022-5223/\$36.00

Copyright (c) 2013 by The American Association for Thoracic Surgery

http://dx.doi.org/10.1016/j.jtcvs.2012.11.071
}

functional aortic annulus (FAA; Figure 1), and the valve cusps. The integrity of both functional components (the cusps and the FAA) is the basis for good valvular function, and alteration in either of these components is frequently associated with alteration in the other. Thus a fundamental principle in AV repair is that both lesions of the cusps and lesions of the FAA should be addressed at the time of valve repair.

In a normal $\mathrm{AV}$, although cusp coaptation relatively constantly reaches the midlevel between the AVJ and the STJ, a certain degree of individual variation exists in the length of coaptation, ranging from 2 to $6 \mathrm{~mm}$. Although a coaptation of $2 \mathrm{~mm}$ is enough to ensure valve competence in an FAA of normal size, longer coaptation may represent some sort of coaptation reserve in the event of FAA dilatation. This anatomic feature, in combination with the individual compliance of the cusp tissues, explains the multiple clinical presentations with regard to the correspondence between the degree of FAA dilatation and the severity of AI. FAA dilatation undoubtedly induces progressive AI; however, significant AI can already be seen with a moderately dilated FAA $(40 \mathrm{~mm})$, whereas some patients have little or no AI even with a severely dilated FAA $(>55 \mathrm{~mm})$. The concept of coaptation reserve, quantified by the coaptation length, is one of the parameters that can provide an estimate of the likely evolution of preoperative or postoperative AI when further dilatation of the FAA is expected.

\section{FUNCTIONAL CLASSIFICATION OF AI}

A functional classification of AI should ideally identify all the contributing lesions of the cusps and the FAA, enable a reconstructive approach to the $\mathrm{AV}$, and help to discriminate patients in whom a durable repair is achievable. We previously described such a classification of $\mathrm{AI}^{1}$ that encompasses all the different causes of AI; provides a common language to be used by surgeons, cardiologists, and echocardiographers; helps the surgeon in choosing repair 


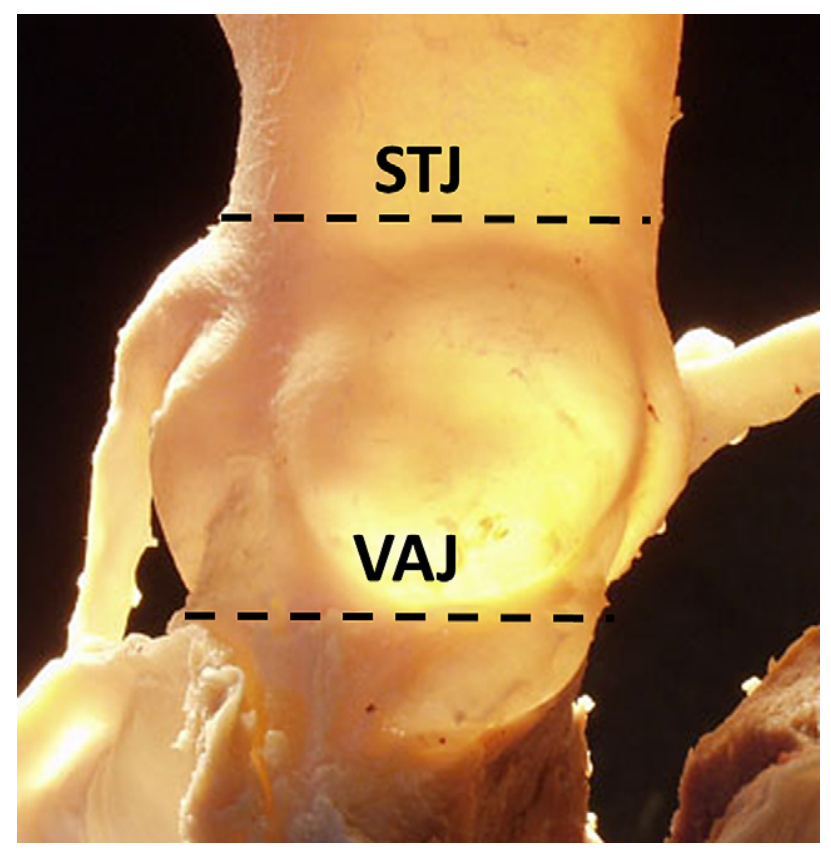

FIGURE 1. Illustration of the functional aortic annulus, composed proximally of the aortoventricular junction $(A V J)$ and distally of the sinotubular junction (STJ).

techniques; and predicts the immediate success and longterm durability of the repair procedure. In this classification, analogous to the mitral valve, type 1 disease is caused by lesions of the FAA in the setting of normal cusp motion, type 2 disease is caused by excessive cusp motion resulting from cusp prolapse, and type 3 disease is caused by restrictive cusp disease.

In this context, 2 important concepts deserve mention. The first notion is that multiple lesions can simultaneously contribute to AI; for example, dilatation of the aortic root or ascending aorta may be associated with alterations in cusp geometry and cusp prolapse. Thus correction of all contributing lesions is critical for successful repair. The second notion is that severe chronic valve insufficiency, as with the mitral valve, can lead to dilatation of the annulus. Thus any correction of cusp pathology in the setting of chronic severe AI should be accompanied by annuloplasty of the FAA, which has 2 components: the proximal AVJ and the distal STJ.

\section{GENERAL PRINCIPLES OF AV REPAIR}

The goal of AV repair is to restore a normal surface of coaptation by restoring normal geometry to the leaflets and FAA while preserving normal mobility of the AV cusps. The mobility of the valve cusps can be viewed as a ratio between the free margin length and the length of the base of cusp implantation, such that:

$$
\text { Cusp mobility } \approx \frac{\text { Free margin length }}{\text { Length of cusp insertion }}
$$

An increase in this ratio leads to greater cusp mobility and may occur because of the presence of excess free margin length or overreduction of length of cusp insertion by annuloplasty (or the choice of a small prosthesis for a valvesparing root replacement procedure). Similarly, a reduction in this ratio leads to reduced cusp mobility or cusp restriction. This is frequently observed in bicuspid AVs, which have a large base of cusp insertion relative to free margin length, as well as in cases of overcorrection of cusp prolapse by reduction of free margin length. This concept also explains the idea that an annuloplasty of the AVJ (reduction of the length of cusp insertion) can improve cusp mobility, reduce the AV gradient, and increase cusp coaptation.

\section{CUSP REPAIR IN TRICUSPID AV}

Cusp prolapse, by definition, occurs as the result of an increase in the free margin length compared with the length of the base of cusp insertion. This leads to a decrease in the height of the prolapsing cusp compared with the normal cusp or cusps. Prolapse correction therefore requires a reduction in free margin length. The critical question thus is how much reduction in the free margin is required to restore cusp height to normal?

Assessment of AV geometry is not easy in the empty and arrested heart. By using 3 commissural traction sutures, the axis of the AV may be oriented towards the surgeon's view. Moreover, by applying a vertical and outward tension on those sutures, the cusps can be stretched, facilitating the comparison between the free margin's height and length. In the case of prolapse of 1 or 2 cusps, the reference height can be taken from the nonprolapsing cusp or cusps. In single-cusp prolapse, a 6-0 polypropylene suture is placed successively in the middle of the 2 nonprolapsing free margins. By applying gentle traction on this stitch and grasping the middle of the prolapsing free margin with a forceps, one can easily compare free margin lengths, and the excess length can be determined objectively. In double-cusp prolapse, a similar stay suture is placed in the middle of the nonprolapsing free margin, and the excess lengths of the 2 prolapsing free margins are successively determined with the same method of comparison.

Prolapse of all 3 cusps is rare in the native AV, but it occasionally can be induced after a valve-sparing procedure. In this situation, the reference used to correct prolapse can be the middle height of the commissures, or it can be determined by an instrument to measure effective cusp height, as previously described by Schäfers and colleagues. ${ }^{2}$ The techniques described for cusp prolapse correction include free margin plication ${ }^{3}$ and free margin resuspension with a running suture of polytetrafluoroethylene. ${ }^{4}$

\section{CUSP REPAIR IN BICUSPID AV}

Regurgitant bicuspid AVs frequently present with dilatation of the aortic annulus, aortic root, or ascending aorta, 
and therefore almost always multiple lesions are responsible for the AI. Cusp morphology in the bicuspid AV can be heterogeneous, and the classification system of Sievers and Schmidtke ${ }^{5}$ provides a useful framework to divide the valves into 2 general types. Type 0 bicuspid AVs are less common, do not contain a median raphe, and have 2 symmetric aortic sinuses, 2 commissures, and a symmetric base of leaflet implantation of the 2 cusps. The mechanism of $\mathrm{AI}$ in this setting is usually prolapse of 1 or both cusps and dilatation of the FAA. The more prevalent type 1 bicuspid AVs have a median raphe on the conjoint cusp and an asymmetric distribution of the aortic sinuses, with a large aortic sinus accompanying a large nonconjoint cusp and 2 smaller cusps fused together with a median raphe. The raphe often attaches to the cusp base in the form of a "pseudocommissure," which has a height lower than that of the true commissures. AI in type 1 valves can be due to a rigid and restrictive raphe associated with small fused cusps, resulting in a triangular coaptation defect. Alternatively, the raphe may be short and nonrestrictive, with well-developed cusps and associated prolapse of the conjoint cusp. Bicuspid $\mathrm{AVs}$ can exist anywhere along a spectrum between type 0 and type $1{ }^{6}$

In type 0 valves, cusp prolapse correction is performed as for tricuspid AVs, with either a nonprolapsing cusp as the reference or restoration of the height of coaptation to the middle height of the commissures. Cusp prolapse is repaired with free margin plication or resuspension, and the often thickened and fibrotic central aspect of the leaflet is resected. In type 1 valves, the median raphe is addressed first. If the raphe is relatively mobile and only mildly thickened and fibrosed, it is preserved and shaved. If the raphe is restrictive or calcified, a parsimonious triangular resection of this tissue is performed. Next, the quantity of remaining cusp tissue is assessed by putting the 2 arms of a 6-0 polypropylene suture on the free margin of the conjoint cusp, on either side of the resected raphe. At this point, lack of cusp restriction and good valve opening are signs of the presence of adequate cusp tissue. The leaflet edges are reapproximated primarily when adequate cusp tissue is present; in the absence of adequate tissue, a triangular autologous treated or bovine pericardial patch is used for cusp restoration. Next, the free margins of both cusps are compared for the presence of any prolapse, which is corrected with free margin plication or resuspension.

Considering the frequency of FAA dilatation in bicuspid $\mathrm{AVs}$, restoration or stabilization of all or part of the FAA must always be considered in association with cusp repair. If STJ dilatation is present, diameter is restored or stabilized with ascending aortic replacement or with a valve-sparing root replacement procedure. In the very frequent case of AVJ dilatation, subcommissural annuloplasty was routinely performed during our early experience; however, the results showed a significant risk of recurrent dilatation with time. ${ }^{7}$
We therefore have modified our approach and have since used valve-sparing reimplantation more liberally, because this technique provides prosthesis-based circumferential annuloplasty of the AVJ. This technique, generally indicated to treat root aneurysms, is actually performed in any bicuspid AV with AVJ dilatation, regardless of the size of the root. In comparison with the subcommissural annuloplasty, reimplantation increases surgical difficulty, but it does not increase postoperative morbidity and does increase the durability of the repair. In the future, to avoid root replacement in patients without root dilatation, specific techniques of AVJ annuloplasty should be developed to provide adequate and durable stabilization of the AVJ in bicuspid $\mathrm{AV}$ repair.

\section{OUTCOME}

Data on the durability of AV repair techniques are currently limited to single-center series that are mostly small to moderate in size, with a follow-up time of 5 to 10 years. Patients undergoing cusp repair with tricuspid AVs (mean age, 58 years; $74 \%$ male; $66 \%$ with associated aortic disease) have a 10 -year freedom from AV reoperation of $89 \%$, and freedom from recurrent AI greater than $2+$ of $86 \%$ in our experience; these freedoms are $92 \%$ and $80 \%$, respectively in other large series. These results are very similar to those reported by others. ${ }^{8}$

Bicuspid AV repair, performed in a slightly younger cohort (mean age, 44 years; $93 \%$ male; $65 \%$ with associated aortic dilatation), leads to excellent survival; $94 \%$ at 10 years in our series, with a freedom from AV reoperation of $81 \%$. For the tricuspid AVs, our results are very similar to the ones reported in other large series. ${ }^{8}$ Valve-sparing root replacement for root and annular stabilization leads to a more durable outcome than is seen with either no annuloplasty or subcommissural annuloplasty alone. ${ }^{7,9,10}$ A consistent finding across our series and another major series of $\mathrm{AV}$ repairs is the low risk of late valve-related morbidity: thromboembolism $(<0.7 \% /$ patient year), bleeding $(<0.3 \% /$ patient year $)$, and endocarditis $(<0.2 \% \text { /patient year })^{8}$

\section{CONCLUSIONS}

$\mathrm{AV}$ repair is a safe and feasible alternative to AV replacement in selected patients with AI with or without aortic dilatation. The results are durable in the midterm, but long-term studies that compare outcome of repair with replacement will better define the role of repair in this population. The low incidence of valve-related morbidity makes valve repair particularly attractive for young patients who want to avoid long-term anticoagulation.

\section{References}

1. Boodhwani M, de Kerchove L, Glineur D, Poncelet A, Rubay J, Astarci P, et al. Repair-oriented classification of aortic insufficiency: impact on surgical techniques and clinical outcomes. J Thorac Cardiovasc Surg. 2009;137:286-94. 
2. Schäfers HJ, Bierbach B, Aicher D. A new approach to the assessment of aortic cusp geometry. J Thorac Cardiovasc Surg. 2006;132:436-8.

3. Boodhwani M, de Kerchove L, Glineur D, El Khoury G. A simple method for the quantification and correction of aortic cusp prolapse by means of free margin plication. J Thorac Cardiovasc Surg. 2010;139:1075-7.

4. Boodhwani M, El Khoury G. Aortic valve repair. Operative techniques in thoracic and cardiovascular surgery. Op Tech Thorac Cardiovasc Surg. 2009;14: 266-80.

5. Sievers HH, Schmidtke C. A classification system for the bicuspid aortic valve from 304 surgical specimens. J Thorac Cardiovasc Surg. 2007;133:1226-33.

6. Boodhwani M, de Kerchove L, Glineur D, Rubay J, Vanoverschelde JL, Noirhomme P, et al. Repair of regurgitant bicuspid aortic valves: a systematic approach. J Thorac Cardiovasc Surg. 2010;140:276-84.e1.
7. de Kerchove L, Boodhwani M, Glineur D, Vandyck M, Vanoverschelde JL, Noirhomme P, et al. Valve sparing-root replacement with the reimplantation technique to increase the durability of bicuspid aortic valve repair. J Thorac Cardiovasc Surg. 2011;142:1430-8.

8. Aicher D, Fries R, Rodionycheva S, Schmidt K, Langer F, Schäfers HJ. Aortic valve repair leads to a low incidence of valve-related complications. Eur J Cardiothorac Surg. 2010;37:127-32.

9. Aicher D, Kunihara T, Abou Issa O, Brittner B, Gräber S, Schäfers HJ. Valve configuration determines long-term results after repair of the bicuspid aortic valve. Circulation. 2011;123:178-85.

10. Alsoufi B, Borger MA, Armstrong S, Maganti M, David TE. Results of valve preservation and repair for bicuspid aortic valve insufficiency. $J$ Heart Valve Dis. 2005;14:752-8; discussion 758-9. 